\title{
The efficacy of radiofrequency ablation in the treatment of pediatric arrhythmia and its effects on serum IL-6 and hs-CRP
}

\author{
CHUNLI LI, LIBO JIA, ZHENZHOU WANG, LING NIU and XINJIANG AN \\ Department of Cardiology, Xuzhou Children's Hospital, Xuzhou, Jiangsu 221000, P.R. China
}

Received May 15, 2017; Accepted August 7, 2017

DOI: $10.3892 / \mathrm{etm} .2017 .4960$

\begin{abstract}
The aim of this study was to investigate the efficacy of radiofrequency ablation in the treatment of pediatric arrhythmia and to assess the changes in serum interleukin-6 (IL-6) and hs-CRP levels after treatment. Hundred and six children with tachyarrhythmia who were admitted to Xuzhou Children's Hospital from November, 2014 to December, 2015 were recruited for study. The efficacies of radiofrequency in the treatment of different types of arrhythmia were analyzed. Successful ablation was found in 104 cases $(98.11 \%)$ and recurrence was found in 7 cases $(6.73 \%)$. Among 62 cases of atrioventricular reentrant tachycardia (AVRT), successful ablation was found in 60 cases $(96.77 \%)$ and recurrence was found in 3 cases $(4.84 \%)$. Among 33 cases of atrioventricular nodal reentrant tachycardia (AVNRT), successful ablation was found in 33 cases $(100 \%)$ and recurrence was found in 2 cases (6.06\%). Among 5 cases of ventricular tachycardia (VT), successful ablation was found in 5 cases $(100 \%)$ and no recurrence was found. Among 4 cases of atrial tachycardia (AT), successful ablation was found in 4 cases (100\%) and recurrence was found in 1 case $(25 \%)$. Among 2 cases of atrial flutter (AFL), successful ablation was found in both (100\%) and recurrence was found in 1 case $(50 \%)$. After operation, the levels of IL- 6 and hs-CRP were increased and were continually increased within $6 \mathrm{~h}$ after operation. The levels of IL-6 and hs-CRP at $24 \mathrm{~h}$ after operation were reduced but still higher than preoperative levels. The duration of radiofrequency and ablation energy were positively correlated with the levels of IL- 6 and hs-CRP, while the number of discharges was not significantly correlated with either. In conclusion, radiofrequency ablation is a safe and effective treatment for pediatric arrhythmia. Postoperative monitoring of IL-6 and hs-CRP levels is conducive to understanding postoperative myocardial injury and inflammatory response.
\end{abstract}

Correspondence to: Dr Xinjiang An, Department of Cardiology, Xuzhou Children's Hospital, 18 Sudi North Road, Xuzhou, Jiangsu 221000, P.R. China

E-mail: anxinjian001@163.com

Key words: radiofrequency ablation, pediatric arrhythmia

\section{Introduction}

Pediatric arrhythmia is a common cardiovascular disease with diverse types and various symptoms observed in pediatric medicine. Common types of pediatric arrhythmias include atrioventricular reentrant tachycardia (AVRT), atrioventricular nodal reentrant tachycardia (AVNRT), ventricular tachycardia (VT), idiopathic VT, cardiac conduction disease and congenital atrioventricular block (1). Among these types of arrhythmias, many cases show no obvious symptoms and have no significant effects on children's life and health. However, some cases of arrhythmia can lead to serious clinical symptoms, with a ventricular rate surpassing 200 beats/min in some patients. Without intervention, the recurrence of arrhythmia can cause persistent myocardial damage, leading to heart expansion or even sudden death (2). In the past, the focus of treatment of pediatric arrhythmia has been the selection of proper antiarrhythmic drugs based on the arrhythmia types. However, this traditional treatment method can only alleviate the symptoms, but cannot achieve radical treatment. In addition, the long-term use of anti-arrhythmic drugs can seriously affect the growth and development of the patient, which limits their clinical application (3). Nowadays, with the continuous development of interventional medicine, radiofrequency ablation has become an alternative to drug therapy in the treatment of a variety of types of pediatric arrhythmias (4). Radiofrequency ablation has even become the preferred method of treatment of many types of arrhythmias.

Radiofrequency ablation is the use of radio frequency to generate and direct thermal energy to destroy the key parts or pathways of the heart that can cause arrhythmia (5), thus eliminating the occurrence of arrhythmia. As such, radiofrequency ablation will inevitably have adverse effects on the myocardium, and even cause myocardial damage (6). Studies have pointed out that myocardial injury and myocardial pathology changes caused by radiofrequency ablation are correlated with an inflammatory response (7). In this study, radiofrequency ablation was used to treat pediatric arrhythmia and the efficacies of those methods were discussed. The changes of serum levels of interleukin-6 (IL-6) and hs-CRP and other inflammatory indicators were also observed before and after operation, to explore the clinical values of the two indicators in postoperative myocardial injury. 


\section{Materials and methods}

General information. A total of 106 children with tachyarrhythmia admitted to Xuzhou Children's Hospital from November, 2014 to December, 2015 were enrolled, including 62 males and 44 females. The ages ranged from 3 years and 10 months to 13 years, with an average age of $8.28 \pm 4.85$ years. The patients diagnosed by the physical examination in our hospital were divided into the following categories: 62 cases of AVRT (including 41 cases of left accessory pathway and 21 cases of right accessory pathway), 33 cases of AVNRT, 5 cases of VT, 4 cases of atrial tachycardia (AT) and 2 cases of atrial flutter (AFL). The normal heart rate and heart rate during arrhythmia were recorded. Heart echocardiography was administered to understand the heart structure and cardiac function of the patients. At least 5 half-lives of the antiarrhythmic drugs were reached or no history of the use of antiarrhythmic drugs was required of the patients before radiofrequency ablation was performed. Patients with comorbidities of congenital heart disease, diabetes, central nervous system disease or liver and kidney disease and patients with a history of acute or chronic infections were excluded from the study. This study was approved by the Ethics Committee of Xuzhou Children's Hospital. Signed written informed consents were obtained from their guardians.

\section{Preparation before surgery}

Preoperative examination. Before surgery, a systematic physical examination was carried out along with all necessary laboratory tests, including routine blood, routine urine, routine fecal, liver function, renal function, cardiac function, coagulation function, syphilis detection, hepatitis B detection and others. Routine 12-lead electrocardiogram, $24 \mathrm{~h}$ dynamic electrocardiogram, tomography and posterior chest exam were also performed.

Preparation before surgery. The patients were fasted for $8 \mathrm{~h}$ before surgery. In addition, $4 \mathrm{~h}$ water deprivation was also performed before surgery. A collar and lead skirt were used to protect the thyroid and gonads.

Non-tracheal intubation intravenous anesthesia using propofol was performed for the patients younger than 12 years, while local anesthesia using lidocaine was performed for the patients older than 12 years.

After anesthesia, coronary sinus mapping electrodes were inserted through left subclavian vein puncture. Right ventricle, right atrium and His bundle mapping electrodes were placed by right femoral vein puncture. After that, the electrophysiological maps of the body surface and the heart were simultaneously recorded.

Since a child's heart is relatively smaller than an adult's heart, a proportionately-sized ablation catheter was selected before surgery. The ablation catheter was positioned in the proper place and measures were taken to ensure the proper ablation energy was applied. More specific operative details were adhered to with reference to guidelines on pediatric radiofrequency ablation by Friedman et al (8). Radiofrequency ablation machines and multi-channel physiological recorders, model LEAD-7000C, were purchased in Sichuan Jinjiang Electronic Science and Technology Co., Ltd. (Chengdu, China).
Ablation of AVRT. Left accessory pathway ablation for AVRT was performed with a small curved electrode catheter. The ablation site is normally on the side of the ventricles, but can be the side of atrium for older patients. Ablation energy temperature was controlled between $50-55^{\circ} \mathrm{C}$, with the power generally between $30-35 \mathrm{~W}$. The ablation energy was adjusted according to the outcomes.

Right accessory pathway ablation for AVRT was performed with a stiffened electrode catheter. A multi-functional sheath was used to improve the stability of the catheter and the ablation site was generally in the right atrioventricular groove. The ablation energy temperature was controlled between $50-55^{\circ} \mathrm{C}$, with the power generally between $40-50 \mathrm{~W}$.

Ablation of AVNRT. Radiofrequency ablation with blocked reentry loop was primarily used to maintain the conduction function of the atrioventricular node in AVNRT. The 7-plus stiffened electrode catheter was used with a multi-functional sheath to improve the stability of the catheter. The ablation energy temperature was controlled at $50^{\circ} \mathrm{C}$, with the power generally between $25-30 \mathrm{~W}$.

VT ablation. Idiopathic ventricular tachycardia (IVT) is the most common type of VT in children. The areas of excitability are most commonly found in the right ventricular outflow tract (RVOT) and the left ventricular septum (ILVT), and less commonly in other inflammatory sites such as the left ventricular outflow tract. For children with suspected RVOT, a $6 \mathrm{~F}$ electrode catheter was generally used. The $6 \mathrm{~F}$ electrode catheter was pushed through the sheath into the right ventricle or outflow tract. The ablation energy temperature was controlled at $50^{\circ} \mathrm{C}$, with the power generally between 30-40 W. For children with suspected ILVT, a 7F electrode catheter was generally used. The ablation energy temperature was controlled at $50^{\circ} \mathrm{C}$, with the power generally between $30-40 \mathrm{~W}$.

AT ablation. Excitatory sequence mapping was primarily used for AT ablation. The ablation catheter was perfused with saline. The ablation energy temperature was controlled at $40^{\circ} \mathrm{C}$, with the power generally between $30-40 \mathrm{~W}$.

AFL ablation. Blocked reentry loop is the key in the success of atrial flutter radiofrequency ablation. In the present study, line-like ablation performed at the lower part of the atrium was the most commonly used method and the $7 \mathrm{~F}$ electrode was the most commonly used catheter. The ablation energy temperature was controlled at $40-45^{\circ} \mathrm{C}$, with the power at $\sim 30 \mathrm{~W}$.

Postoperative treatment. The patients received ECG monitoring generally for $72 \mathrm{~h}$ immediately after surgery. Patients without major complications were transferred to a general ward. Any postoperative complications, including postoperative arrhythmia, pericardial tamponade, wound hematoma and pneumothorax, were monitored during the observation period, with convalescent care provided. A routine 12-lead electrocardiogram, tomography and posterior chest exam were performed again before discharge.

Blood samples collection and examination. The venous blood samples from all patients were collected and examined before surgery. The venous blood samples from all patients were also collected and examined at 6 and $24 \mathrm{~h}$ after surgery. 
Table I. The efficacy of radiofrequency ablation in the treatment of different types of arrhythmia.

\begin{tabular}{lccl}
\hline Types & $\begin{array}{c}\text { Cases } \\
\mathrm{n}\end{array}$ & $\begin{array}{c}\text { Success cases } \\
(\%)\end{array}$ & $\begin{array}{c}\text { Failure } \\
\text { cases }(\%)\end{array}$ \\
\hline AVRT & 62 & $60(96.77)$ & $2(3.23)$ \\
AVNRT & 33 & $33(100)$ & $0(0)$ \\
VT & 5 & $5(100)$ & $0(0)$ \\
AT & 4 & $4(100)$ & $0(0)$ \\
AFL & 2 & $2(100)$ & $0(0)$ \\
Total & 106 & $104(98.11)$ & $2(1.89)$ \\
\hline
\end{tabular}

AVRT, atrioventricular reentrant tachycardia; AVNRT, atrioventricular nodal reentrant tachycardia; VT, ventricular tachycardia; AT, atrial tachycardia; AFL, atrial flutter.

Table II. Recurrence rates of different types of arrhythmia.

\begin{tabular}{lccr}
\hline Type & $\begin{array}{c}\text { Cases with } \\
\text { recurrence }(\%)\end{array}$ & $\begin{array}{c}\text { Cases without } \\
\text { recurrence }(\%)\end{array}$ & $\begin{array}{r}\text { Total } \\
\text { (cases) }\end{array}$ \\
\hline AVRT & $3(5.00)$ & $57(95.00)$ & 60 \\
AVNRT & $2(6.06)$ & $31(93.94)$ & 33 \\
VT & $0(0.00)$ & $5(100)$ & 5 \\
AT & $1(25.00)$ & $3(75.00)$ & 4 \\
AFL & $1(50.00)$ & $1(50.00)$ & 2 \\
Total (cases) & $7(6.73)$ & $97(93.27)$ & 104 \\
$\chi^{2 \text { a }}$ & $8.76^{\mathrm{a}}$ & & \\
P-value & $>0.05$ & & \\
\hline
\end{tabular}

${ }^{a}$ Chi-square value indicates that the difference in recurrence rates between the different types of arrhythmia was not significant. AVRT, atrioventricular reentrant tachycardia; AVNRT, atrioventricular nodal reentrant tachycardia; VT, ventricular tachycardia; AT, atrial tachycardia; AFL, atrial flutter.

A $6 \mathrm{ml}$ blood sample was also collected from each patient each time-point. An enzyme-linked immunosorbent assay (ELISA) kit (Beijing Biolab Science and Technology Co. Ltd., Beijing, China) was used to detect the level of IL-6. The detection of hs-CRP was performed using a Hitachi 7600 automatic biochemical analyzer (Hitachi Co., Tokyo, Japan).

Follow-up. The patients were asked to return for follow-up with a routine 12-lead electrocardiogram and tomography at 7 days, 1 month, 6 months and 1 year after surgery. Information on the living conditions of the patients was obtained by regularly contacting the patient via phone.

Statistical methods. SPSS 19.0 statistical software (IBM, New York, NY, USA) was used to analyze the data. The measurement data were expressed as mean \pm standard deviation and was analyzed using the independent samples t-test; count data were expressed as a percentage (\%) and was analyzed using the Chi-square test. The correlation test was
Table III. Levels of serum inflammation indicators before and after surgery.

\begin{tabular}{lcc}
\hline Time-points & IL-6 $(\mathrm{pg} / \mathrm{ml})$ & hs-CRP $(\mathrm{mg} / \mathrm{l})$ \\
\hline Before surgery & $6.12 \pm 1.12$ & $8.26 \pm 1.47$ \\
After surgery & $8.38 \pm 2.21^{\mathrm{a}, \mathrm{b}}$ & $10.54 \pm 2.13^{\mathrm{a}, \mathrm{b}}$ \\
6 h after surgery & $10.97 \pm 2.86^{\mathrm{a}}$ & $13.76 \pm 3.31^{\mathrm{a}}$ \\
24 h after surgery & $8.87 \pm 2.67^{\mathrm{a}, \mathrm{b}}$ & $11.43 \pm 2.59^{\mathrm{a}, \mathrm{b}}$ \\
\hline
\end{tabular}

${ }^{\mathrm{a} C}$ Compared with the level before surgery, $\mathrm{p}<0.05$; ${ }^{\mathrm{b}}$ compared with the level at $6 \mathrm{~h}$ after surgery, $\mathrm{p}<0.05$. IL-6, interleukin- 6 .

performed using Pearson's correlation analysis for data with a normal distribution and Spearman's correlation analysis was used for data with a non-normal distribution. $\mathrm{p}<0.05$ was considered to be statistically significant.

\section{Results}

The efficacy of radiofrequency ablation. Hundred and six children with tachyarrhythmia were selected for study. Successful ablation was found in 104 cases (98.11\%), while 2 cases failed (1.89\%). Recurrence was found in 7 cases, for a recurrence rate of $6.73 \%$.

The efficacy of radiofrequency ablation in the treatment of different types of arrhythmia. Among the 62 cases of AVRT, successful ablation was found in 60 cases $(96.77 \%)$, while 2 cases failed (3.23\%). Among the 33 cases of AVNRT, successful ablation was found in 33 cases (100\%). Among the 5 cases of VT, successful ablation was found in 5 cases $(100 \%)$. Among the 4 cases of AT, successful ablation was found in 4 cases $(100 \%)$. Among the 2 cases of AFL, successful ablation was found in 2 cases (100\%) (Table I).

Recurrence rate of different types of arrhythmia. Among the 106 patients, recurrence was found in 7 cases, for a total recurrence rate of $6.73 \%$. During follow-up, the average recurrence time was 7 months. Among the 62 cases of AVRT, recurrence was found in 3 cases. Among the 33 cases of AVNRT, recurrence was found in 2 cases $(6.06 \%)$. Among the 5 cases of VT, no recurrence was found $(0.00 \%)$. Among the 4 cases of AT, recurrence was found in 1 case $(25.00 \%)$. Among the 2 cases of AFL, recurrence was found in 1 case $(50.00 \%)$. There was no significant difference in recurrence rates between the different types of arrhythmia $(\mathrm{p}>0.05)$ (Table II).

Levels of serum inflammation indicators before and after surgery. After the operation, the serum levels of IL-6 and hs-CRP were increased and were continually increased within $6 \mathrm{~h}$ after operation. The levels of IL- 6 and hs-CRP at $24 \mathrm{~h}$ after operation were reduced but still higher than the preoperative levels (Table III and Figs. 1 and 2).

Correlations between radiofrequency ablation parameters and inflammatory indicators. The correlations between the duration of radiofrequency, ablation energy and the number 
Table IV. Correlations of the duration of radiofrequency, ablation energy and the number of discharges with the inflammatory indicators.

\begin{tabular}{|c|c|c|c|c|c|c|}
\hline & \multicolumn{2}{|c|}{ Duration of radiofrequency (s) } & \multicolumn{2}{|c|}{ Ablation energy $(\mathrm{J})$} & \multicolumn{2}{|c|}{ No. of discharges (times) } \\
\hline & r-value & P-value & r-value & P-value & r-value & P-value \\
\hline IL-6 & ${ }^{\mathrm{a}} 0.503$ & $<0.05$ & ${ }^{\mathrm{a}} 0.453$ & $<0.05$ & 0.191 & 0.008 \\
\hline Hs-CRP & ${ }^{\mathrm{a}} 0.374$ & $<0.05$ & ${ }^{\mathrm{a}} 0.327$ & $<0.05$ & 0.065 & 0.44 \\
\hline
\end{tabular}

${ }^{\mathrm{a} C}$ Correlation coefficient $(\mathrm{r})$ between factors was significant $(\mathrm{p}<0.05)$. IL-6, interleukin-6.

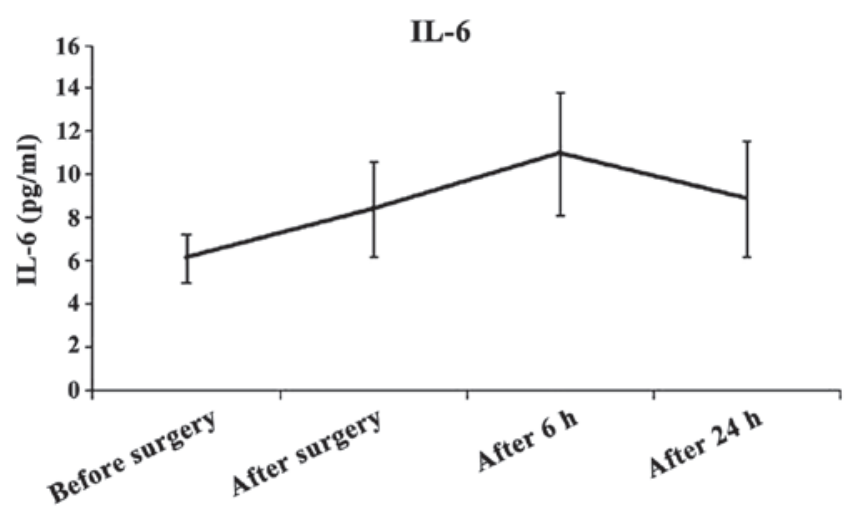

Figure 1. The changes in interleukin-6 (IL-6) levels after operation. Enzyme-linked immunosorbent assay (ELISA) results showed that the level of IL- 6 was increased and was continually increased within $6 \mathrm{~h}$ after operation. The level of IL-6 at $24 \mathrm{~h}$ after the operation was still higher than the preoperative levels.

of discharges with the inflammatory indicators were analyzed. The results showed that the duration of radiofrequency and ablation energy were positively correlated with the levels of IL- 6 and hs-CRP, while the number of discharges was not significantly correlated with the levels of IL-6 or hs-CRP (Table IV).

Complications. Among the 106 cases, complete atrioventricular block was found in 1 case after radiofrequency ablation. No serious complications or deaths were observed among the patients over the course of the study.

\section{Discussion}

Tachyarrhythmia is a common disease with different symptoms observed in pediatric cardiovascular medicine. Tachyarrhythmia shows no obvious symptoms in some patients and can only be detected by physical examination. While some patients showed paroxysmal palpitations, chest tightness and even syncope has been known to occur in severe cases. Without proper treatment, the recurrence of tachyarrhythmia can cause changes in the body's hemodynamics and possible secondary expansion and reduced cardiac function, and may finally lead to the development of cardiomyopathy (9). These diseases can not only seriously affect the physical and mental health of children, but also bring heavy economic burden to the patient's family. In the past, anti-arrhythmic drugs and cardioversion were widely used in the treatment of arrhythmia, but

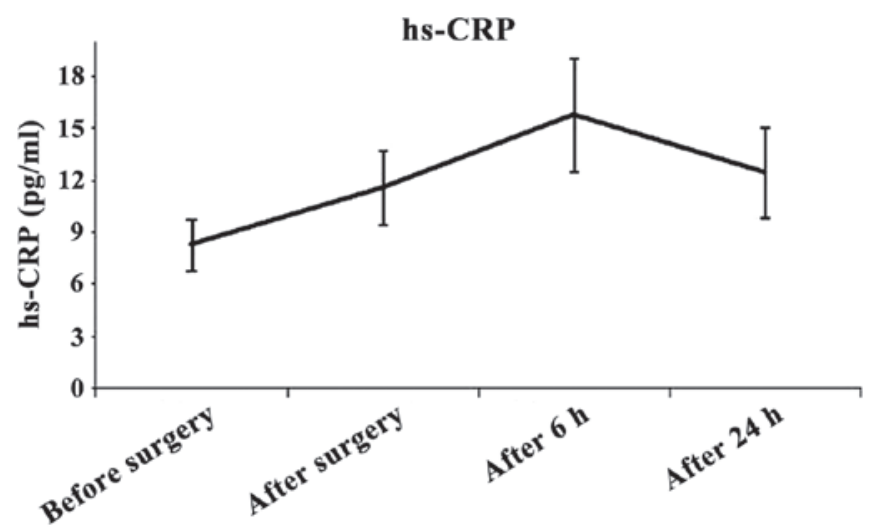

Figure 2. The changes in Hs-CRP levels after operation. Biochemical analysis showed that the level of Hs-CRP was increased and was continually increased within $6 \mathrm{~h}$ after operation. The level of Hs-CRP at $24 \mathrm{~h}$ after operation was still higher than the preoperative levels.

antiarrhythmic drugs can trigger adverse side effects among children (3). In addition, cardioversion cannot prevent the recurrence of arrhythmia. Therefore, those two methods both have limitations (10).

In the 1990s, the pathophysiology of tachyarrhythmia was gradually elucidated. Heart electrophysiological examination, which can be used to determine the origins of these arrhythmias, provides the basis for the application of radiofrequency ablation in the treatment of tachyarrhythmia. In 1989, radiofrequency ablation was first used to treat children with Wolff-Parkinson-White syndrome; in 1991, radiofrequency ablation was then used in the treatment of pediatric tachyarrhythmia (11). With the development of radiofrequency ablation and the continuous improvements of its techniques, radiofrequency ablation has become widely used in the treatment of pediatric tachyarrhythmia. Due to the advantages of better safety, reliable treatment effect and reduced risk of trauma (12), this method has now been accepted by most medical institutions in the world $(13,14)$. Radiofrequency ablation has replaced antiarrhythmic drugs, cardioversion and surgery to become the main therapy in the treatment of pediatric tachyarrhythmia (15).

In this study, successful ablation was observed in 104 out of 106 cases of tachyarrhythmia, accounting for $98.11 \%$ of all cases, while recurrence was found in 7 cases, accounting for $6.73 \%$, indicating an overall satisfactory treatment efficacy. Specifically, 62 cases of AVRT (58.49\%), 33 cases of AVNRT (31.13\%), 5 cases of VT (4.72\%), 4 cases of AT (3.77\%) and 
2 cases of AFL (1.89\%) constituted the 106 cases. Most of the patients (95 cases) had AVRT or AVNRT, accounting for $89.62 \%$ of cases, which is consistent with previous studies that showed supraventricular tachycardia to be the most common type of pediatric tachyarrhythmia, accounting for $~ 90 \%$ of all cases of pediatric arrhythmias (16). Among the 62 cases of AVRT patients, 60 cases showed successful ablation and 2 cases failed, for a success rate of $96.77 \%$, indicating a satisfactory treatment efficacy.

The 3 cases of recurrence, which were all found in the left-sided accessory pathways, were due to the lack of a coronary sinus electrode to serve as the reference electrode and the difference in anatomical structure between the two sides, which resulted in incomplete ablation. In this study, the ablations in patients with AVNRT were all successful and the success rate was higher than previously reported (95.7-97\%) (4), which may be due to the relatively small sample size of the present study. The ablations in patients with VT were all successful and the success rate was higher than previously reported (17). Likewise, a possible explanation is the small sample size of our study. None of the patients with AT of AFL had a failure of the treatment, but their recurrence rate was comparatively high. Further studies with larger number of samples would help to increase the frequencies of diagnoses of tachyarrhythmia types and improve the evaluation of the efficacies of their treatment.

At present, the most commonly reported complications of radiofrequency ablation include atrioventricular block, acute pericardial tamponade, peripheral vascular injury and bleeding, pneumothorax and thromboembolism (18). In this study, 1 out of 106 patients showed a slight degree of atrioventricular block. This occurrence had no adverse effects on the child's life and health and regular follow-up care was performed as for all cases. Overall, the results indicate that radiofrequency ablation is safe but attention to the occurrence of the aforementioned complications and timely treatment are necessary.

After radiofrequency ablation, the pathological changes of the ablation site are mainly coagulation necrosis, which is due to the thermal effects caused by radio frequency energy (19). After treatment, acute inflammatory responses, such as the rapid exudation of monocytes and neutrophils, can be observed around the necrotic tissue, indicating significant myocardial injury in the affected necrotic area (20). In human body,Hs-CRP is an acute-phase reaction protein with high sensitivity, and the increase of Hs-CRP usually indicates an inflammatory reaction in the body (21). IL-6 is an interleukin that functions as a cytokine protein and can be secreted by macrophages or other cells after injury. IL-6 is an important inflammatory factor in the human body. IL- 6 can not only induce an acute inflammatory response, but can also activate a variety of immune cells to promote their differentiation and activation, which in turn increases the cell damage caused by inflammation (22).

In this study, IL-6 and hs-CRP levels were increased immediately after radiofrequency ablation. The levels reached a peak at $6 \mathrm{~h}$ after operation, and were still higher than normal levels at $24 \mathrm{~h}$ after operation. This suggests that inflammation occurred in the early stages after radiofrequency ablation. IL-6 and hs-CRP levels after the operation were positively correlated with the duration of radiofrequency and the time of ablation. This suggests that extended durations of radiofrequency ablation can increase the thermal effect on the myocardium and cause a higher degree of myocardial damage (23). In addition, greater ablation energy results in proportionately increased organization of the interface temperature and current density, thereby impacting the heart with more severe thermal injury of the myocardium (24). Therefore, tactical control of the radiofrequency duration and the rational use of radiofrequency energy levels may help reduce myocardial injury and inflammatory response.

In conclusion, radiofrequency ablation is a safe and effective therapy in the treatment of pediatric arrhythmia. The postoperative monitoring of the changes of IL-6 and hs-CRP can be used to understand the damage of the postoperative myocardial tissue and the inflammatory reaction, which provides the basis for the application and control of reasonable radiofrequency energy and duration during surgery.

\section{References}

1. Blaufox AD, Paul T and Saul JP: Radiofrequency catheter ablation in small children: Relationship of complications to application dose. Pacing Clin Electrophysiol 27: 224-229, 2004.

2. Blaufox AD, Felix GL and Saul JP; Pediatric Catheter Ablation Registry: Radiofrequency catheter ablation in infants $\leq 18$ months old: when is it done and how do they fare?: Short-term data from the pediatric ablation registry. Circulation 104: 2803-2808, 2001.

3. Wazni O, Martin DO, Marrouche NF, Shaaraoui M, Chung MK, Almahameed S, Schweikert RA, Saliba WI and Natale A: C reactive protein concentration and recurrence of atrial fibrillation after electrical cardioversion. Heart 91: 1303-1305, 2005.

4. Sakaguchi H, Miyazaki A, Yamamoto M, Kurosaki K, Ohuchi H, Satomi K, Suyama K and Yamada O: Clinical characteristics of focal atrial tachycardias arising from the atrial appendages during childhood. Pacing Clin Electrophysiol 34: 177-184, 2011.

5. Morady F: Radio-frequency ablation as treatment for cardiac arrhythmias. N Engl J Med 340: 534-544, 1999.

6. Alaiti MA, Maroo A and Edel TB: Troponin levels after cardiac electrophysiology procedures: Review of the literature. Pacing Clin Electrophysiol 32: 800-810, 2009.

7. Conway DS, Buggins P, Hughes E and Lip GY: Prognostic significance of raised plasma levels of interleukin- 6 and C-reactive protein in atrial fibrillation. Am Heart J 148: 462-466, 2004.

8. Friedman RA, Walsh EP, Silka MJ, Calkins H, Stevenson WG, Rhodes LA, Deal BJ, Wolff GS, Demaso DR, Hanisch D, et al; NASPE Expert Consensus Conference: Radiofrequency catheter ablation in children with and without congenital heart disease. Report of the writing committee. North American Society of Pacing and Electrophysiology. Pacing Clin Electrophysiol 25: 1000-1017, 2002

9. Allessie M, Ausma J and Schotten U: Electrical, contractile and structural remodeling during atrial fibrillation. Cardiovasc Res 54: 230-246, 2002.

10. Tieleman RG, De Langen C, Van Gelder IC, de Kam PJ, Grandjean J, Bel KJ, Wijffels MC, Allessie MA and Crijns HJ: Verapamil reduces tachycardia-induced electrical remodeling of the atria. Circulation 95: 1945-1953, 1997.

11. Walsh EP and Saul JP: Transcatheter ablation for pediatric tachyarrhythmias using radiofrequency electrical energy. Pediatr Ann 20: 386-392, 388-392, 1991.

12. Van Hare GF, Javitz H, Carmelli D, Saul JP, Tanel RE, Fischbach PS, Kanter RJ, Schaffer M, Dunnigan A, Colan S, et al; Pediatric Electrophysiology Society: Prospective assessment after pediatric cardiac ablation: Demographics, medical profiles, and initial outcomes. J Cardiovasc Electrophysiol 15: 759-770, 2004.

13. Borger van der Burg AE, de Groot NM, van Erven L, Bootsma M, van der Wall EE and Schalij MJ: Long-term follow-up after radiofrequency catheter ablation of ventricular tachycardia: A successful approach? J Cardiovasc Electrophysiol 13: 417-423, 2002.

14. Raungratanaamporn O, Bhuripanyo K, Sriratanasathavorn C and Chotinaiwattarakul C: Radiofrequency catheter ablation for various tachyarrhythmias: Experience in the Bangkok Heart Institute. J Med Assoc Thai 86 (Suppl 1): S105-S109, 2003. 
15. Lee PC, Hwang B, Chen SA, Tai CG, Chen YJ, Chiang CE and Meng CC: The results of radiofrequency catheter ablation of supraventricular tachycardia in children. Pacing Clin Electrophysiol 30: 655-661, 2007.

16. Joung B, Lee M, Sung JH, Kim JY, Ahn S and Kim S: Pediatric radiofrequency catheter ablation: Sedation methods and success, complication and recurrence rates. Circ J 70: 278-284, 2006.

17. Baksiene D, Sileikiene R, Sileikis V, Kazakevicius T, Zabiela V, Zebiene $\mathrm{M}$ and Puodziukynas A: Idiopathic ventricular tachycardia in children: Curative therapy with radiofrequency ablation. Medicina (Kaunas) 43: 803-807, 2007.

18. Lee SJ and Schueller WC: Tachycardias in infants, children and adolescents: Safety and effectiveness of radiofrequency catheter ablation. Cardiology 94: 44-51, 2000.

19. Wren C: Catheter ablation in paediatric arrhythmias. Arch Dis Child 81: 102-104, 1999.

20. Dudar TE and Jain RK: Differential response of normal and tumor microcirculation to hyperthermia. Cancer Res 44 605-612, 1984.
21. Rifai N and Ridker PM: High-sensitivity C-reactive protein: A novel and promising marker of coronary heart disease. Clin Chem 47: 403-411, 2001.

22. Cruickshank AM, Oldroyd KG and Cobbe SM: Serum interleukin-6 in suspected myocardial infarction. Lancet 343: 974-981, 1994.

23. Hirose H, Kato K, Suzuki O, Yoshida T, Oguri M, Yajima K, Hibino T and Yokoi K: Diagnostic accuracy of cardiac markers for myocardial damage after radiofrequency catheter ablation. J Interv Card Electrophysiol 16: 169-174, 2006.

24. Emkanjoo Z, Mottadayen M, Givtaj N, Alasti M, Arya A, Haghjoo M, Fazelifar AF, Alizadeh A and Sadr-Ameli MA: Evaluation of post-radiofrequency myocardial injury by measuring cardiac troponin I levels. Int J Cardiol 117: 173-177, 2007. 